\title{
Predictive value of preoperative comprehensive evaluation on the efficacy of HoLEP
}

\author{
Xinglin Chen ${ }^{1 \#}$, Qiuhong Man ${ }^{2 \#}$, Xiyi Wei ${ }^{1 \#}$, Xiaohan Ren ${ }^{1}$, Guangyao Li ${ }^{1}$, Zhongwen Lu ${ }^{1}$, Xu Zhang ${ }^{1}$, \\ Chao Qin ${ }^{1}$
}

${ }^{1}$ Department of Urology, the First Affiliated Hospital of Nanjing Medical University, Nanjing, China; ${ }^{2}$ Department of Clinical Laboratory, Shanghai Fourth People's Hospital Affiliated to School of Medicine, Tongji University, Shanghai, China

Contributions: (I) Conception and design: C Qin, X Chen; (II) Administrative support: C Qin; (III) Provision of study materials or patients: X Chen, Q Man, X Wei; (IV) Collection and assembly of data: X Chen, X Zhang; (V) Data analysis and interpretation: X Ren, G Li, Z Lu; (VI) Manuscript writing: All authors; (VII) Final approval of manuscript: All authors.

\#These authors contributed equally to this work.

Correspondence to: Chao Qin. Department of Urology, the First Affiliated Hospital of Nanjing Medical University, Nanjing 210009 , China.

Email: qinchao@njmu.edu.cn.

\begin{abstract}
Background: With the aging of the population, prostate hyperplasia is more and more perplexing to the old men. This study aimed to evaluate the predictive value of international prostatic symptom score-voiding/ storage score ratio (IPSS-V/S), urodynamic examination, intravesical prostatic protrusion (IPP) and residual urine volume (RUV) on the efficacy of holmium laser enucleation of the prostate (HoLEP) before operation. Methods: Ninety-six patients with benign prostatic hyperplasia (BPH) were enrolled in this study. Each patient was diagnosed as BPH and completed all the evaluations as described above before the same urologist surgeon performed HoLEP. Three months later, another IPSS questionnaire would be finished by each patient to assess the efficacy of the operation. According to the results of IPSS after operation, the group with significant effect and the group with poor effect were divided. Then compare the difference of preoperative examination results between the two groups.

Results: In single factor analysis, the differences of the age, voiding symptom score, V/S, RUV, Qmax and IPP between the two groups were statistically significant $(\mathrm{P}<0.05)$. While there was no significant difference in IPSS score and score of urine storage period. In the logistic regression analysis, the age of patients, V/S, RUV, IPP and Qmax can establish a good prediction model. In this model, the younger the patients are, the less the RUV is, the larger the V/S and IPP are, the more likely they are to achieve good efficacy of HoLEP.

Conclusions: In conclusion, HoLEP was more effective in BPH patients with younger age, more IPP, less RUV, less Qmax and those whose IPSS score was mainly based on voiding symptoms.
\end{abstract}

Keywords: Benign prostatic hyperplasia (BPH); international prostatic symptom score-voiding/storage score ratio (IPSS-V/S); intravesical prostatic protrusion (IPP); residual urine volume (RUV); predictive value; holmium laser enucleation of the prostate (HoLEP)

Submitted Feb 03, 2020. Accepted for publication Jun 30, 2020.

doi: 10.21037/tau-20-504

View this article at: http://dx.doi.org/10.21037/tau-20-504

\section{Introduction}

There is a positive correlation between the incidence of $\mathrm{BPH}$ and age (1). With the increase of age, the volume of prostate in old men increases by about $2.0-2.5 \%$ /year $(2,3)$. The incidence of $\mathrm{BPH}$ was $40-49$ years $(8 \%), 60-69$ years
(50\%), 90-99 years (80\%) (1). Clinically, the lower urinary tract symptoms (LUTS) associated with BPH perplex about $20 \%$ of 40 years old men and about $50 \%$ of 80 years old men $(4,5)$. Although the vast majority of patients can take drugs to effectively improve symptoms (6), with the 
development of minimally invasive prostate surgery, more and more patients tend to surgical treatment (7). For many years, transurethral resection of prostate (TURP) is the standard treatment when the drug cannot control the symptoms of benign prostatic hyperplasia (BPH) and decades of clinical practice has fully proved the safety and effectiveness of TURP (8-10). However, holmium laser enucleation of the prostate (HoLEP) may become the new standard treatment taking the place of TURP nowadays for its better outcomes and less complications (11). Meanwhile many Meta-analysis reports give support to advantages of HoLEP $(12,13)$. Despite all this, quite a lot of the patients are not satisfied with the results of operation (14). In order to explore the factors that affect the effect of operation, we conducted a retrospective study retrospective analysis of the clinical data of $98 \mathrm{BPH}$ patients admitted to the First Affiliated Hospital of Nanjing Medical University from December 2018 to December 2019.

We present the following article in accordance with the STROBE reporting checklist (available at http://dx.doi. org/10.21037/tau-20-504).

\section{Methods}

The study was conducted in accordance with the Declaration of Helsinki (as revised in 2013) and the Harmonized Tripartite Guideline for Good Clinical Practice from the International Conference on Harmonization. This study was reviewed and approved by Ethics Committee of Nanjing Medical university (approval number 2019-SR125). All patients enrolled completed the informed consent form.

\section{Study population}

$96 \mathrm{BPH}$ patients were admitted to the First Affiliated Hospital of Nanjing Medical University from December 2018 to December 2019, aged $72.72 \pm 7.94$ years. Inclusion criteria: ultrasound examination confirmed hyperplasia of prostate, and repeated urinary retention or frequency, urgency and nocturia which could not be controlled satisfactorily by drugs. Exclusion criteria: prostate cancer, urothelial cancer, neurogenic urinary system disease and patients who cannot complete the postoperative follow-up.

All patients were evaluated with IPSS-V/S, urodynamic study (UDS), and ultrasonography before operation. The IPP value was measured by transabdominal ultrasound. In the supine position, when the bladder is properly filled
(100-200 mL), a 3.5 MHz transabdominal ultrasound probe is used to scan the transverse section and longitudinal sagittal plane. Firstly, the transverse diameter, anterior posterior diameter and upper and lower diameter of the prostate are measured, and then the vertical distance from the highest point of the prostate protruding into the bladder to the bladder neck is measured as the length of IPP through the median sagittal plane.

\section{Operative method \& follow-up}

Under general anesthesia, the patients were performed holmium laser enucleation of the prostate by the same doctor. During the whole operation, attention should be paid to the protection of seminal vesicle gland and complete hemostasis. After complete removal of the hyperplastic glands, clear out all the tissues by morcellator. After careful observation of the wound without obvious active bleeding, catheter was placed, and $40 \mathrm{~mL}$ air bag was compressed to avoid bleeding. After the operation, the bladder was flushed continuously, and $20 \mathrm{~mL}$ gas was drawn out from the air bag on the second day to prevent the urethra from necrosis. On the 3rd to 4th day after operation, after observing the color of the bladder washing liquid, stop the bladder washing and remove the catheter. After 1-2 days of unobstructed urination, the patients were discharged from the hospital.

The patient was asked to come to the hospital three months after the operation, the IPSS and quality of life (QOL) questionnaire was followed up. QOL less than or equal to 2 points after operation is regarded as generally satisfactory. If the IPSS score is improved by more than $50 \%$, or the postoperative IPSS score is less than 7, and the QOL score is generally satisfactory, the operation effect is considered satisfactory, otherwise, the operation effect is considered unsatisfactory. The difference of preoperative examination results between the group with satisfactory and the group with poor efficacy was compared.

\section{Statistical analysis}

Normal data is represented by mean \pm standard deviation, non-normal distribution data by percentile, median (Q1-Q3). The significances of the differences between two groups were evaluated with Student's t-test for the means and standard deviation. The nonparametric test is used between two independent samples that do not obey the normal distribution data; the counting data is expressed by percentage, and the Pearson $\chi 2$ test is used for comparison 
Table 1 Clinical characteristics and single factor analysis of the study patients

\begin{tabular}{|c|c|c|c|c|}
\hline & Total & Successful & Unsuccessful & $P$ value \\
\hline Age & $72.72 \pm 7.94$ & $71.00 \pm 7.20$ & $76.89 \pm 8.23$ & 0.002 \\
\hline IPSS (points) & $23.47 \pm 3.44$ & $23.84 \pm 3.46$ & $22.57 \pm 3.26$ & 0.101 \\
\hline Voiding (points) & $14.29 \pm 2.74$ & $14.81 \pm 2.33$ & $13.04 \pm 3.25$ & 0.012 \\
\hline $\mathrm{V} / \mathrm{S}$ & $1.63(1.40-1.86)$ & $1.65(1.47-1.86)$ & $1.35(1.02-1.94)$ & 0.037 \\
\hline Qmax (mL/s) & $7(5-8)$ & $6(4-7)$ & $8(7-9)$ & $<0.001$ \\
\hline IPP (mm) & $4.0(0-5.0)$ & $5(0-6)$ & $0(0-5)$ & 0.007 \\
\hline RUV (mL) & $116.04 \pm 20.44$ & $110.44 \pm 17.66$ & $129.64 \pm 20.63$ & $<0.001$ \\
\hline
\end{tabular}

Values are expressed as mean \pm standard deviation, number, or median (Q1-Q3).

between groups. Odds ratios (ORs) were presented with $95 \%$ confidence intervals (CIs) and $\mathrm{P}$ values less than 0.05 was considered statistically significant. Adjusted ORs with $95 \%$ CIs and $\mathrm{P}$ values were calculated using logistic regression examination. The nomogram of clinical prediction model was drawn by $\mathrm{R}$ software. All statistical procedures were performed using the Statistical Package for the Social Science (SPSS Inc, Chicago, Illinois, USA) version 23.0. and $\mathrm{R}$ software (R-3.6.2 for Windows).

\section{Results}

The preoperative IPSS-V/S score was $23.47 \pm 3.44$, including $14.29 \pm 2.74$ in voiding and $9(8-10)$ in storage, V/S:1.63 (1.40-1.86), 7 (5-8) mL/s in Qmax, $4.0(0-5.0) \mathrm{mm}$ in IPP, $116.04 \pm 20.44 \mathrm{~mL}$ in RUV.

All the 98 patients successfully completed the HoLEP operation. The anesthesia was satisfactory during the operation. During the enucleation, the bleeding was less, and the hemostasis was complete. There was no uncontrollable massive bleeding. The average time required for enucleation of hyperplastic prostate tissue is about 28 minutes.

The IPSS score of 96 patients after operation was $5.38 \pm 1.97$, which was statistically different from that before operation (23.47 \pm 3.44$)$. Sixty-eight patients $(70.33 \%)$ in the satisfactory group, $71.00 \pm 7.20$ years old, $23.84 \pm 3.46$ points in IPSS, $14.81 \pm 2.33$ points in voiding symptoms, 9 (810) points in storage symptoms, $1.65(1.47-1.86)$ in V/S, 6 (4-7) $\mathrm{mL} / \mathrm{s}$ in Qmax, 5 (0-6) $\mathrm{mm}$ in IPP, $110.44 \pm 17.66 \mathrm{~mL}$ in RUV. Meanwhile 28 patients (29.17\%) in the poor group,
$76.89 \pm 8.23$ years old, $22.57 \pm 3.26$ points in IPSS, points in voiding symptoms was $13.04 \pm 3.25$, storage symptoms score was 9 (8-12), V/S: 1.35 (1.02-1.94), Qmax: 8 (7-9) mL/s, IPP: 0 (0-5) mm, RUV: $129.64 \pm 20.63 \mathrm{~mL}$.

The age, IPSS score, voiding symptom score and RUV of the two groups were tested by independent sample t-test, and the differences among age, voiding symptom score and RUV were statistically significant $(\mathrm{P}<0.05)$. Between the two groups, the score of storage period, V/S, Qmax and IPP were tested by nonparametric test, and the differences among V/S, Qmax and IPP groups were statistically significant (Table 1).

V/S is the sum of the voiding scores to the sum of the storage scores, so there is a certain correlation between the voiding score and the V/S ratio. Compared with the voiding score, the V/S ratio can better reflect whether the lower urinary tract symptoms are mainly in the voiding stage or the storage stage, for voiding score only can reflect the severity of symptoms during micturition. The large V/S ratio can better explain that the lower urinary tract symptoms are mainly in the voiding period, we think that the V/S ratio is more suitable for multiple factor analysis than the voiding period score. On the other hand, there is an inevitable relationship between voiding and V/S. It is obviously biased to incorporate both into the regression prediction model, because there is a collinearity between them. It also supports our view that Model 3 of logistic regression is better than Model 1 and Model 2.

In Model 1, we tried to incorporate all factors that were statistically relevant to the outcome of the surgery in the univariate analysis into the regression model. But 
Table 2 Multiple logistic regression analysis of factors associated with success rate of HoLEP

\begin{tabular}{|c|c|c|c|c|c|c|c|c|c|}
\hline & \multicolumn{3}{|c|}{ Model 1} & \multicolumn{3}{|c|}{ Model 2} & \multicolumn{3}{|c|}{ Model 3} \\
\hline Age & 0.84 & $0.72-0.98$ & 0.03 & 0.84 & $0.72-0.98$ & 0.03 & 0.85 & $0.73-0.98$ & 0.02 \\
\hline Qmax & 0.44 & $0.18-0.88$ & 0.02 & 0.40 & $0.18-0.88$ & 0.02 & 0.46 & $0.23-0.91$ & 0.03 \\
\hline IPP & 1.37 & $1.03-1.81$ & 0.03 & 1.36 & $1.04-1.80$ & 0.03 & 1.31 & $1.01-1.70$ & 0.04 \\
\hline V/S & 0.85 & $0.09-7.90$ & 0.88 & - & - & - & 6.20 & $1.48-32.45$ & 0.01 \\
\hline Voiding & 1.72 & $1.13-2.61$ & 0.01 & 1.68 & $1.24-2.29$ & 0.00 & - & - & - \\
\hline
\end{tabular}

OR, odds ratio; $95 \% \mathrm{Cl}, 95 \%$ confidence intervals.

the prediction effect of the model is not ideal, RUV and V/S are difficult to be included as statistically significant variables. In model 2, we chose not to include V/S ratio in the prediction model, and RUV also lacked statistical significance. In Model 3, the age of patients, V/S, RUV, IPP and Qmax were included in the logistic regression model and all factors included are statistically significant. It was found that the age of patients, RUV and Qmax were the risk factors of unsatisfactory surgical results. The older the patients, the more RUV and Qmax were, the greater the possibility of poor surgical results was. V/S ratio and IPP are the protective factors of satisfactory operation effect. The higher V/S ratio (mainly voiding symptoms) and IPP are, the more likely the operation is to succeed (Table 2).

Based on multi-factor analysis, we use $\mathrm{R}$ software to draw nomogram of clinical prediction model, the model as shown in the figure is obtained. According to the score axis at the top, the scores of each variable are obtained, and then the total score is calculated. Finally, the success rate is obtained on the prediction axis at the bottom according to the total score. Comparatively speaking, IPP has little effect on the total score. However, it is not difficult to see that other variables included in the nomogram have an important impact on the success rate of surgery (Figure 1).

\section{Discussion}

There have been a lot of reports on whether the parameters of BPH patients, such as age, residual urine volume, IPSS score, can predict the effect of BPH surgery (15-17), and they also formed the criteria for the success of the operation: the improvement of IPSS score is more than $50 \%$ or postoperative IPSS score is less than 7 ; the improvement of maximum urine flow rate is more than
$50 \%$ or postoperative Qmax is more than $15 \mathrm{~mL} / \mathrm{s}$; the reduction of residual urine volume is more than $50 \%$ or more than $60 \mathrm{~mL}$ (18). In decades of years' work, we found that in the first period of time after operation, patients often had the condition of dysuria caused by inflammation and edema or other reasons and urinary incontinence, which would get better in the next few weeks. If the patient's symptoms do not improve within a few weeks, the possibility of subsequent improvement is also relatively small. Therefore, we chose three months after the operation for follow-up. Because IPSS score is simple and low cost, its effectiveness has been fully confirmed in previous studies, and the influence of LUTS symptoms on life is subjective. In this study, IPSS combined with QOL score is selected as the evaluation standard of efficacy, and it is concluded that $\mathrm{BPH}$ patients who are younger, whose preoperative IPSS scores are mainly urinary symptoms, who have larger IPP, less RUV, and smaller Qmax are more likely to obtain satisfactory HoLEP results. We believe that the key to the effect of HoLEP is to identify the cause of LUTS and the early intervention before the secondary damage of bladder function.

Benign prostatic hyperplasia (BPH) is only a histological diagnosis (19), while clinical BPH refers to a series of symptoms of bladder outlet obstruction (BOO) caused by benign hyperplasia of prostate tissue, i.e., benign prostatic obstruction (BPO), which may be followed by clinical manifestations of bladder and renal function damage (20). Clinically, patients with BPH tend to see doctors for LUTS symptoms. A large multinational study shows that $90 \%$ of men aged 50-80 have latent LUTS symptoms, and a large part of them have both voiding and storage symptoms (21). Chun et. point out LUTS symptoms of overactive bladder $(\mathrm{OAB})$ and underactive bladder (UAB) are easily confused 


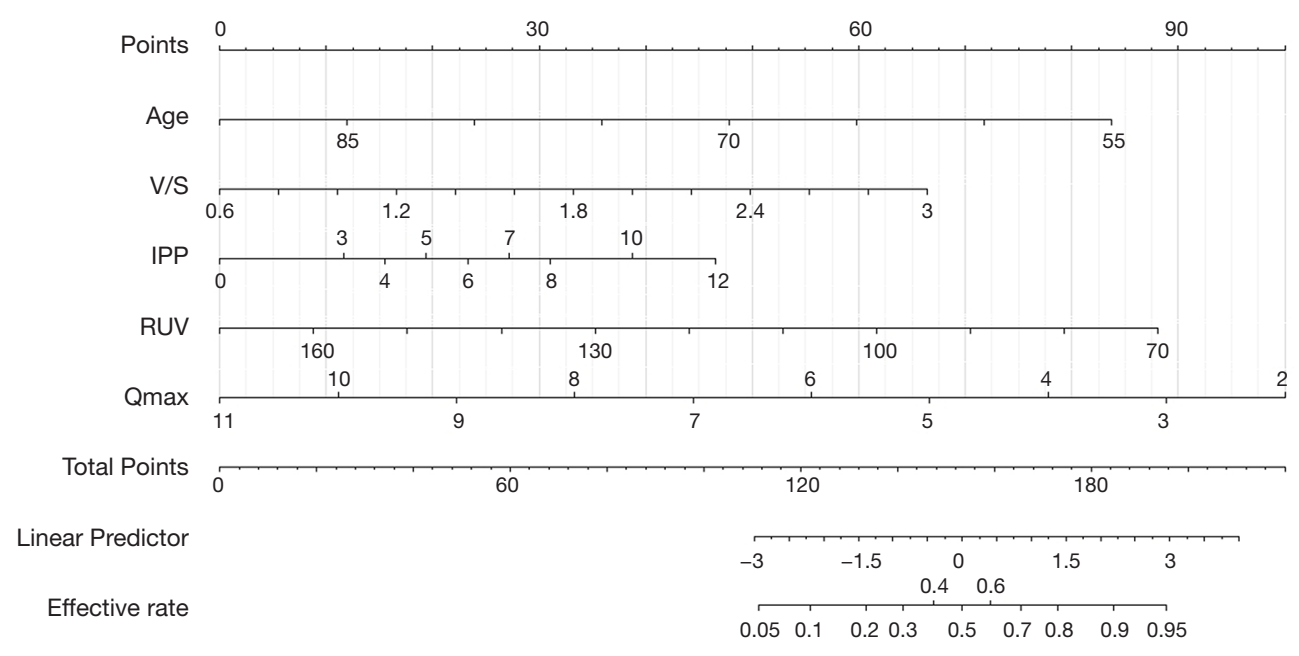

Figure 1 Nomogram of different factors associated with success rate of HoLEP.

with BPH (22).

$\mathrm{OAB}$ is mainly seen in the spontaneous dysfunction of bladder function caused by detrusor overactivity (DO) and detrusor hyperactivity and inadequate contractility (DHIC), as well as the secondary impairment of bladder outlet obstruction caused by $\mathrm{BPH}$, bladder neck dysfunction and dysrelaxation of urinary sphincter (23). Both DO and DHIC are positively correlated with age (23), which is consistent with the epidemiology of BPH. In addition, the vast majority of patients with $\mathrm{BPH}$ have $\mathrm{DO}$, but nearly half of DO patients do not have BPH $(24,25)$, which brings great difficulties in differential diagnosis. Because the symptoms of OAB and LUTS in BPH are the same, and the prevalence also increases with age, and the imaging prostate enlargement (BPE) is very common, some LUTS patients were misdiagnosed as $\mathrm{BPH}$, and received unnecessary and invalid drugs and surgical treatment (26). In this study, we found that patients with BPH who's preoperative IPSS score is mainly urinary symptoms and whose Qmax is smaller have better results of HoLEP. Patients with significant voiding symptoms are more likely to have LUTS symptoms due to BPH than overactive bladder. In this study, although there are statistical differences in the voiding score and $\mathrm{V} /$ $S$ ratio between the group with satisfactory effect and the group with poor effect, we still think that the V/S ratio is more significant. IPSS score is the score of patients' subjective feelings, and the large V/S ratio compared with the high voiding score indicates that LUTS symptoms of this patient are mainly in the voiding period. This was also verified in logistic regression analysis.

It is very common for the elderly to have $\mathrm{UAB}$, which is mainly manifested by detrusor underactivity (DU). In particular, the prevalence of chronic urinary retention is higher in elderly people with chronic diseases, such as diabetes, chronic heart disease, neurological diseases and so on (27). More than two-thirds of elderly inpatients with urinary incontinence suffer from DU (28). At the same time, the long-term obstruction of bladder outlet caused by $\mathrm{BPH}$ will also change the structure and function of bladder wall and urinary retention or excessive residual urine caused by bladder outlet obstruction may also cause decreased detrusor contractility (29). Therefore, it is very difficult to distinguish the primary DU from that secondary to bladder outlet obstruction. The biggest pressure of detrusor in 81 cases of failure of operation reported by Djvan et al. is generally smaller, which is believed to be caused by over extension and reduction of contractile force of bladder (30). Liao and Kuo believed that the total prostate volume (TPV) greater than $40 \mathrm{~mL}$ is more likely to have bladder outlet obstruction (22), but it has been previously reported that LUTS symptoms are not necessarily related to prostate volume, and smaller prostate may also cause significant symptoms (31). Bladder outlet obstruction depends more on the location of hyperplasia than the size of prostate (32). Urodynamic examination also confirmed that the obstruction effect of urethral distortion on urinary flow was more obvious than that of urethral global constriction $(33,34)$. In this study, we chose IPP to measure the effect of BPH on LUTS symptoms and found that HoLEP was better in patients with large IPP, which was consistent with the previous consensus. We believe that patients with large IPP are more likely to have LUTS symptoms due to bladder 
outlet obstruction caused by BPH. Even if the secondary detrusor contractility is low, the detrusor contractility may recover after TURP operation to solve the obstruction. The patients with younger age and less RUV are more likely to avoid the serious damage of bladder function caused by the long-term obstruction of bladder outlet due to the short course of disease and the non over stretching of bladder, and the HoLEP operation is more effective.

Previous studies have shown that the continuous enlargement of the prostate will lead to continuous progress of lower urinary tract symptoms (35). With the prolongation of life expectancy, $\mathrm{BPH}$, a benign disease that plagues many old men, deserves attention. Although the prostate volume can be reduced by $25 \%$ and LUTS symptoms can be significantly improved by the combination of $\alpha$ blocker and 5- $\alpha$ reductase inhibitor (36), some patients still need HoLEP and other surgical treatment because of the ineffective drug treatment or other reasons. Because there are many reasons for LUTS symptoms, how to accurately predict the success rate of surgery and avoid ineffective surgical treatment has been the focus of many scholars. Through the retrospective analysis of the clinical data of $98 \mathrm{BPH}$ patients admitted to the First Affiliated Hospital of Nanjing Medical University, it was found that HoLEP was more effective in BPH patients with younger age, more symptoms during voiding, less maximum urinary flow rate (Qmax), greater intravesical prostatic protrusion (IPP) and less residual urine volume (RUV). However, due to the small sample size and the lack of further verification of the model, the research results need to be further explored.

\section{Conclusions}

Through this study, we have come to the conclusion that the older patients may have poor surgical effect due to the long course of disease leading to the secondary injury of detrusor and bladder, in addition, some patients may not or not be completely caused by the obstruction of bladder outlet caused by prostate hyperplasia and hypertrophy, so the simple HoLEP operation is difficult to achieve satisfactory results. We believe that it will be helpful to avoid the ineffectiveness of HoLEP operation to clarify the real cause of lower urinary tract symptoms and operate before the secondary injury of detrusor of bladder caused by the long course of disease.

\section{Acknowledgments}

Funding: None.

\section{Footnote}

Reporting Checklist: The authors have completed the STROBE reporting checklist. Available at http://dx. doi. org/10. 21037/tau-20-504

Data Sharing Statement: Available at http://dx. doi. org/10. 21037/tau-20-504

Peer Review File: Available at http://dx. doi. org/10.21037/ tau-20-504

Conflicts of Interest: All authors have completed the ICMJE uniform disclosure form (available at http://dx. doi. org/10. 21037/tau-20-504). The authors have no conflicts of interest to declare.

Ethical Statement: The authors are accountable for all aspects of the work in ensuring that questions related to the accuracy or integrity of any part of the work are appropriately investigated and resolved. The study was conducted in accordance with the Declaration of Helsinki (as revised in 2013) and the Harmonized Tripartite Guideline for Good Clinical Practice from the International Conference on Harmonization. This study was reviewed and approved by Ethics Committee of Nanjing Medical university (approval number 2019-SR-125). All patients enrolled completed the informed consent form.

Open Access Statement: This is an Open Access article distributed in accordance with the Creative Commons Attribution-NonCommercial-NoDerivs 4.0 International License (CC BY-NC-ND 4.0), which permits the noncommercial replication and distribution of the article with the strict proviso that no changes or edits are made and the original work is properly cited (including links to both the formal publication through the relevant DOI and the license). See: https://creativecommons.org/licenses/by-nc-nd/4.0/.

\section{References}

1. Berry SJ, Coffey DS, Walsh PC, et al. The development 
of human benign prostatic hyperplasia with age. J Urol 1984;132:474-9.

2. Bosch JL, Tilling K, Bohnen AM, et al. Establishing normal reference ranges for prostate volume change with age in the population-based Krimpen-study: prediction of future prostate volume in individual men. Prostate 2007;67:1816-24.

3. Loeb S, Kettermann A, Carter HB, et al. Prostate volume changes over time: results from the Baltimore Longitudinal Study of Aging. J Urol 2009;182:1458-62.

4. Speakman M, Kirby R, Doyle S, et al. Burden of male lower urinary tract symptoms (LUTS) suggestive of benign prostatic hyperplasia $(\mathrm{BPH})$ - focus on the UK. BJU Int 2015;115:508-19.

5. Wei JT, Calhoun E, Jacobsen SJ. Urologic diseases in America project: benign prostatic hyperplasia. J Urol 2005;173:1256-61.

6. Fwu CW, Eggers PW, Kaplan SA, et al. Long-term effects of doxazosin, finasteride and combination therapy on quality of life in men with benign prostatic hyperplasia. J Urol 2013;190:187-93.

7. Ferretti M, Phillips J. Prostatectomy for benign prostate disease: open, laparoscopic and robotic techniques. Can J Urol 2015;22 Suppl 1:60-6.

8. Umari P, Fossati N, Gandaglia G, et al. Robotic Assisted Simple Prostatectomy versus Holmium Laser Enucleation of the Prostate for Lower Urinary Tract Symptoms in Patients with Large Volume Prostate: A Comparative Analysis from a High Volume Center. J Urol 2017;197:1108-14.

9. Autorino R, Zargar H, Mariano MB, et al. Perioperative Outcomes of Robotic and Laparoscopic Simple Prostatectomy: A European-American Multi-institutional Analysis. Eur Urol 2015;68:86-94.

10. Roehrborn CG, Gange SN, Shore ND, et al. The prostatic urethral lift for the treatment of lower urinary tract symptoms associated with prostate enlargement due to benign prostatic hyperplasia: the L.I.F.T. Study. J Urol 2013;190:2161-7.

11. Vincent MW, Gilling PJ. HoLEP has come of age. World J Urol 2015;33:487-93.

12. Ahyai SA, Gilling P, Kaplan SA, et al. Meta-analysis of functional outcomes and complications following transurethral procedures for lower urinary tract symptoms resulting from benign prostatic enlargement. Eur Urol 2010;58:384-97.

13. Yin L, Teng J, Huang CJ, et al. Holmium laser enucleation of the prostate versus transurethral resection of the prostate: a systematic review and meta-analysis of randomized controlled trials. J Endourol 2013;27:604-11.

14. Emberton M, Neal DE, Black N, et al. The effect of prostatectomy on symptom severity and quality of life. Br J Urol 1996;77:233-47.

15. Abrams PH, Dunn M, George N. Urodynamic findings in chronic retention of urine and their relevance to results of surgery. Br Med J 1978;2:1258-60.

16. George NJ, Feneley RC, Roberts JB. Identification of the poor risk patient with "prostatism" and detrusor failure. $\mathrm{Br}$ J Urol 1986;58:290-5.

17. Radomski SB, Herschorn S, Naglie G. Acute urinary retention in men: a comparison of voiding and nonvoiding patients after prostatectomy. J Urol 1995;153:685-8.

18. Javlé P, Jenkins SA, Machin DG, et al. Grading of benign prostatic obstruction can predict the outcome of transurethral prostatectomy. J Urol 1998;160:1713-7.

19. Abrams P, Cardozo L, Fall M, et al. The standardisation of terminology in lower urinary tract function: report from the standardisation sub-committee of the International Continence Society. Urology 2003;61:37-49.

20. Gratzke C, Bachmann A, Descazeaud A, et al. EAU Guidelines on the Assessment of Non-neurogenic Male Lower Urinary Tract Symptoms including Benign Prostatic Obstruction. Eur Urol 2015;67:1099-109.

21. Rosen R, Altwein J, Boyle P, et al. Lower urinary tract symptoms and male sexual dysfunction: the multinational survey of the aging male (MSAM-7). Eur Urol 2003;44:637-49.

22. Liao CH, Kuo HC. Current consensus and controversy on the treatment of male lower urinary tract symptoms/ benign prostatic hyperplasia. Ci Ji Yi Xue Za Zhi 2017;29:1-5.

23. Wein AJ. Bladder outlet obstruction--an overview. Adv Exp Med Biol 1995;385:3-5; discussion 75-9.

24. Knutson T, Edlund C, Fall M, et al. BPH with coexisting overactive bladder dysfunction--an everyday urological dilemma. Neurourol Urodyn 2001;20:237-47.

25. Hyman MJ, Groutz A, Blaivas JG. Detrusor instability in men: correlation of lower urinary tract symptoms with urodynamic findings. J Urol 2001;166:550-2; discussion 553 .

26. Temml C, Heidler S, Ponholzer A, et al. Prevalence of the overactive bladder syndrome by applying the International Continence Society definition. Eur Urol 2005;48:622-7.

27. Ueda T, Yoshimura N, Yoshida O. Diabetic cystopathy: relationship to autonomic neuropathy detected by 
sympathetic skin response. J Urol 1997;157:580-4.

28. Resnick NM, Yalla SV, Laurino E. The pathophysiology of urinary incontinence among institutionalized elderly persons. N Engl J Med 1989;320:1-7.

29. Taylor JA, 3rd, Kuchel GA. Detrusor underactivity: Clinical features and pathogenesis of an underdiagnosed geriatric condition. J Am Geriatr Soc 2006;54:1920-32.

30. Djavan B, Madersbacher S, Klingler C, et al. Urodynamic assessment of patients with acute urinary retention: is treatment failure after prostatectomy predictable? J Urol 1997;158:1829-33.

31. Hirayama A, Samma S, Fujimoto K, et al. Comparison of parameters to determine the cause of urinary disturbance in men with prostate volume less than 20 milliliters. Int J Urol 2002;9:554-9; discussion 560.

32. Luo GC, Foo KT, Kuo T, et al. Diagnosis of prostate adenoma and the relationship between the site of prostate

Cite this article as: Chen X, Man Q, Wei X, Ren X, Li G, Lu Z, Zhang X, Qin C. Predictive value of preoperative comprehensive evaluation on the efficacy of HoLEP. Transl Androl Urol 2020;9(4):1603-1610. doi: 10.21037/tau-20-504 adenoma and bladder outlet obstruction. Singapore Med J 2013;54:482-6.

33. Foo KT. Pathophysiology of clinical benign prostatic hyperplasia. Asian J Urol 2017;4:152-7.

34. Foo KT. Solving the benign prostatic hyperplasia puzzle. Asian J Urol 2016;3:6-9.

35. Bosch JL, Bangma CH, Groeneveld FP, et al. The longterm relationship between a real change in prostate volume and a significant change in lower urinary tract symptom severity in population-based men: the Krimpen study. Eur Urol 2008;53:819-25; discussion 825-7.

36. Kaplan SA, Roehrborn CG, McConnell JD, et al. Longterm treatment with finasteride results in a clinically significant reduction in total prostate volume compared to placebo over the full range of baseline prostate sizes in men enrolled in the MTOPS trial. J Urol 2008;180:10302; discussion 1032-3. 